http://dx.doi.org/10.32929/2446-8355.2020v29n2p169-182

\title{
REPETIBILIDADE E SELEÇÃO INDIRETA EM PROGÊNIES DE AÇAIZEIROS PROVENIENTES DE ANAJÁS, PARÁ
}

\section{Gilberto Ken Iti Yokomizo ${ }^{1 *}$, João Tomé de Farias Neto $^{2}$, Maria do Socorro Padilha de Oliveira $^{3}$}

\footnotetext{
1* Pesquisador, Doutor, Genética e Melhoramento de Plantas, Embrapa Amapá, Macapá, AP, Brasil. E-mail do autor correspondente: gilberto.yokomizo@embrapa.br

${ }^{2}$ Pesquisador, Doutor, Genética e Melhoramento de Plantas, Embrapa Amazônia Oriental, Belém, PA, Brasil.

${ }^{3}$ Pesquisadora, Doutora, Genética e Melhoramento de Plantas, Embrapa Amazônia Oriental, Belém, PA, Brasil.
}

RESUMO: O presente trabalho teve como principal objetivo avaliar a repetibilidade em progênies oriundas de áreas ribeirinhas de Anajás e cultivadas num campo experimental em Tomé-Açu, Pará, visando identificar as mais estáveis, para fins de recomendação posterior após seleção. O delineamento experimental utilizado foi blocos casualizados, com trinta progênies, três repetições e cinco plantas por parcela. As características avaliadas foram: peso médio do cacho (PMC); peso total de frutos (PTF); peso médio do cacho vazio (PMCV); tamanho médio da ráquis/cacho (TMR); peso médio de 100 frutos (PCF); número total de cachos (NTC) e; peso total do cacho (PTC). Os resultados da análise de variância mostram que a interação GxA foi mais importante, havendo dificuldades na seleção de materiais superiores e da necessidade de maior número de avaliações. As principais conclusões foram que as diferenças genéticas existentes nas progênies interagem de forma diferenciada com os fatores ambientais. Todas as características relacionadas a peso apresentaram herdabilidades e repetibilidades altas, facilitando uma seleção futura com estas características, exceto em TMR e NTC, necessitando de um grande número de avaliações dificultando os trabalhos de seleção. Com as correlações PTC, pode substituir TMR e NTC de forma indireta. Recomenda-se somente realizar uma seleção para esta população quando se alcançar o número de medições determinados pela análise de repetibilidade.

Palavras-chave: Euterpe oleracea. Biometria. Melhoramento de plantas. Seleção genética.

\section{REPEATABILITY AND INDIRECT SELECTION IN PROGENIES OF ASSAI FROM ANAJÁS, PARÁ}

ABSTRACT: The present work was be principal objective to evaluate the repeatability in progenies from riverside areas of Anajás and cultivated in an experimental field in Tomé-Açu, Pará, seeking to identify the most stable, for later recommendation after selection. The experimental design was a randomized block design with thirty progenies, three replications and five plants per plot. The evaluated characteristics were: bunch average weight (PMC); total fruit weight (PTF); average weight of empty bunch (PMCV); average size of the stem/bunch (TMR); average weight of 100 fruits (PCF); total number of clusters (NTC) and; total weight of the bunch (PTC). The results of the variance analysis show that the GxA 
interaction was more important, with difficulties in selecting superior materials and the need for more evaluations. The main conclusions were that genetic differences in progenies interact differently with environmental factors. All characteristics related to weight presented high heritability and repeatability, facilitating a future selection with these characteristics, except in TMR and NTC, requiring a large number of evaluations making selection difficult. With the PTC correlations, the TMR and NTC can be replaced indirectly. It is recommended to make a selection for this population only when the number of measurements determined by repeatability analysis is reached.

Key words: Euterpe oleracea. Biometry. Plant breeding. Genetic selection.

\section{INTRODUÇÃO}

O açaizeiro (Euterpe oleracea) tem sua importância em fornecer frutos para consumo de sua polpa, também de palmitos, e é uma espécie endêmica da Amazônia, onde a colheita é realizada tradicionalmente de forma extrativa. O Estado do Pará apresenta a maior produção e consumo nacional, contendo grandes maciços de açaizeiros representando diversificadas populações naturais presentes em áreas de várzeas. Na última estimativa publicada do Instituto Brasileiro de Geografia e Estatística IBGE (2017), a safra de 2017 alcançou um total de 219.885 toneladas, arrecadando $\mathrm{R} \$ 596,8$ milhões. A produção extrativa brasileira concentra-se principalmente nos estados da Região Norte, com os Estados do Pará e Amazonas respondendo juntos por $87,5 \%$ desta produção, destacando-se o município de Limoeiro do Ajú, Pará, que sozinho produziu 18,2\% deste volume total.

O consumo crescente tanto dentro do país como internacional, gera uma nova dinâmica do mercado do açaí, cujo extrativismo e o manejo de açaizais em várzea não produz o suficiente para atender à demanda, conduzindo ao processo de cultivo do açaizeiro em terra firme, cujo sistema de produção tem evoluído, adotando-se recomendações técnicas de espaçamento, adubação, irrigação e plantas selecionadas (NOGUEIRA et al., 2013). A adoção de novas tecnologias, principalmente do manejo e plantas geneticamente superiores, no sistema de produção de açaí-fruto refletindo em aumento de produtividade de frutos têm contribuído para a melhoria do benefício socioeconômico de toda a cadeia produtiva (NOGUEIRA; SANTANA, 2016).

Neste processo de melhoramento genético em espécies perenes, a etapa de avaliação é a mais onerosa e demorada, sendo preciso conhecer a estabilidade de produção, semelhante ao citado por Chia et al. (2009). Assim, a aplicação de metodologias eficientes que aumentem a acurácia no processo seletivo, é de extrema importância (FARIAS NETO et al., 2008), conseguindo-se estimar parâmetros genéticos em testes de progênies de espécies arbóreas visando predizer os ganhos originários de estratégias aplicadas no melhoramento genético e, dessa forma, orientar na escolha do método de seleção mais adequado (MAIA et al., 2009).

Entretanto o açaizeiro apresenta como principais comportamentos biológicos, o ciclo reprodutivo longo, diferentes expressões das características ao longo de vários anos, heterogeneidade em precocidade, perfilhamento e longevidade, dentre outros que trazem algumas dificuldades em predizer os valores genéticos, gerando baixa repetibilidade de suas 
características, e refletindo em maior dispêndio de esforços e custos com a condução de experimentos por vários ciclos. Em vista disso, no processo de seleção de um genótipo superior espera-se que sua superioridade se mantenha e, esse bom desempenho poderá ser comprovado pelo coeficiente de repetibilidade (NEVES et al., 2010), buscando-se o valor máximo que a herdabilidade no sentido amplo pode atingir (LUSH, 2008; CRUZ et al., 2012). Onde a repetibilidade é definida como a correlação entre as médias das parcelas experimentais obtidas em sucessivas avaliações (médias sucessivas) de um único genótipo. Contudo é conveniente ressaltar que em açaizeiro estudos de repetibilidade em características de produtividade de frutos são escassos, tendo-se somente o de Oliveira e Fernandes (2001).

Com base nestas informações o objetivo deste trabalho foi avaliar a repetibilidade de produção em genótipos de açaizeiros nas condições do município de Anajás, Pará, visando identificar as mais estáveis, para fins de recomendação posterior após seleção.

\section{MATERIAL E MÉTODOS}

O estudo foi realizado na área experimental da Embrapa Amazônia Oriental no município de Tomé-Açu, no Nordeste paraense, com topografia plana e solo do tipo latossolo amarelo de textura argilosa, com altitude de $45 \mathrm{~m}$, localizada aproximadamente entre as latitudes as $01^{\circ} 24^{\prime} 46,14^{\prime \prime}$ e $01^{\circ} 28^{\prime} 4,11^{\prime \prime}$ de latitude sul, e $48^{\circ} 20^{\prime} 4,60^{\prime \prime}$ e $48^{\circ} 20^{\prime} 31,84^{\prime \prime}$ de longitude oeste de Greenwich. Possuindo clima quente e úmido, ajustando-se ao tipo climático Ami, da classificação de Köppen, caracterizado como chuvoso, porém com pequena estação seca. A média anual de precipitação pluviométrica é em torno de $2.300 \mathrm{~mm}$. A umidade relativa do ar na região apresenta pouca oscilação ao longo do ano, variando entre 81 e $89 \%$ (valores médios anuais) e a temperatura média do ar é de $26{ }^{\circ} \mathrm{C}$ (VALENTE et al., 2014).

As mudas foram produzidas partindo de sementes coletadas de matrizes nativas de polinização aberta em áreas ribeirinhas no município de Anajás, PA em maio de 2004. Essa região tem como principal característica a produção de frutos em período considerado como entressafra no estado, ou seja, no primeiro semestre. Na seleção fenotípica das árvores em condições naturais utilizaram-se como critérios de seleção o número de cacho por planta, tamanho do cacho, presença de perfilhos e estado fitossanitário das plantas. A pré-germinação das sementes, sem quebra de dormência, foi efetuada com o acondicionamento destas em sacos de plástico transparente fechados, misturadas com serragem fina (pó de serra), curtida e umedecida, na proporção de uma parte de semente para duas partes de serragem, até o máximo de $1 \mathrm{~kg}$ de sementes. Essa mistura foi mantida em condições ambientais (QUEIROZ et al., 2001). O teste de progênies foi estabelecido em março de 2005, usando o delineamento experimental blocos casualizados, com trinta tratamentos (progênies), três repetições e cinco plantas por parcela no espaçamento de $6 \mathrm{~m}$ x $4 \mathrm{~m}$, além de uma linha de bordadura externa ao experimento.

As características avaliadas entre os anos de 2010 a 2012 foram: peso médio do cacho (PMC), em gramas; peso total de frutos (PTF), em gramas; peso médio do cacho vazio (PMCV), em gramas; tamanho médio da ráquis/cacho (TMR), em centímetros; peso médio de 
100 frutos (PCF), em gramas; número total de cachos (NTC) e; peso total do cacho (PTC), obtido por PMC x NTC.

A análise de variância, com nível de significância de 5\%, baseou-se no seguinte modelo, proposto por Cruz et al. (2012): Yijk $=\mathrm{m}+\mathrm{Gi}+\mathrm{Bj}+\mathrm{Ak}+\mathrm{GAik}+$ Eijk; sendo: Yijk: efeito do i-ésimo genótipo no k-ésimo Ano no j-ésimo Bloco, m: média geral dos genótipos; Gi: efeito do genótipo i; Bj: efeito do bloco j; Ak: efeito do ano k; GAik: efeito da interação do genótipo i no ano k; Eijk: erro experimental aleatório. Após a análise de variância para as características avaliadas, as médias dos genótipos foram agrupadas pelo agrupamento de médias de Scott-Knott, a 5\% de probabilidade.

Para se obter as estimativas dos coeficientes de repetibilidade ( $\hat{\rho}$ ) foram empregados cinco diferentes métodos: análise de variância (ANOVA), utilizando o modelo com dois fatores de variação (genótipos e anos de avaliação), análise dos componentes principais com base na matriz de correlações (CPCOR) e de covariâncias (CPCOV), e análise estrutural com base na matriz de correlações (AECOR) e de covariância (AECOV), segundo a metodologia descrita por Cruz et al. (2012). Foram obtidas também as estimativas do número de avaliações (y) necessárias para predizer o valor real dos genótipos a partir de valores estabelecidos para o coeficiente de determinação $\left(\mathrm{R}^{2}\right)$. Todas estas análises foram realizadas com auxílio do programa Genes (CRUZ, 2013).

\section{RESULTADOS E DISCUSSÃO}

Na Tabela 1 referente ao resumo da análise de variância com os quadrados médios e a significância para o teste $F$, foram observadas diferenças significativas entre as progênies $(\mathrm{G})$ somente para TMR e NTC, indicando haver variabilidade genotípica entre os diferentes acessos e a possibilidade de existência de materiais superiores, enquanto que nas demais características não foram obtidas diferenças, diferentemente para PTC e PTF citado por Oliveira e Fernandes (2001) onde existiram diferenças.

$\mathrm{Na}$ fonte de variação anos (A) na análise de variância (Tabela 1), não foram verificadas diferenças significativas, ou seja, no local de experimentação, as diferenças de temperatura, UR do ar e precipitação (Tabela 2) apesar de apresentarem variações não foram suficientes para causarem desigualdade entre as progênies em todas as características avaliadas, aspecto muito interessante, demonstrando estabilidade ambiental, o que é difícil de ocorrer.

Apesar de haver efeito genotípico apenas para TR e NTC e não haver para anos, pode-se observar na Tabela 1 que a interação GxA foi significativa em todas as características avaliadas, indicando que os genótipos interagiram de forma diferenciada com as condições ambientais de cada ano. 
Tabela 1. Resumo da análise de variância para as características peso médio do cacho (PMC), em gramas; peso total de frutos (PTF), em gramas; peso médio do cacho vazio (PMCV), em gramas; tamanho médio da ráquis/cacho (TMR), em centímetros; peso médio de 100 frutos (PCF), em gramas; número total de cachos (NTC) e; peso total do cacho (PTC) em 30 progênies (G) de açaizeiros em quatro anos (A). Summary of variance analysis for the characteristics bunch average weight (PMC); total fruit weight (PTF); average weight of empty bunch (PMCV); average size of the stem/bunch (TMR); average weight of 100 fruits $(P C F)$; total number of clusters (NTC) and; total weight of the bunch (PTC) in 30 progenies $(G)$ of assai palm trees in four years $(A)$.

\begin{tabular}{|c|c|c|c|c|c|c|c|c|}
\hline $\mathrm{FV}$ & GL & PMC & PTF & PMCV & TMR & $\mathrm{PCF}$ & NTC & PTC \\
\hline Blocos & 2 & 858467,4 & 110310102,3 & 191630,1 & 184,3 & 111,2 & 32,2 & 360447630,0 \\
\hline G & 29 & $329237,0^{\mathrm{ns}}$ & $75162813,8^{\mathrm{ns}}$ & $24859,2^{\mathrm{ns}}$ & $39,2 * *$ & $553,6^{\mathrm{ns}}$ & $12,8 * *$ & $153418709,3^{\mathrm{ns}}$ \\
\hline A & 3 & $52994283,2^{\mathrm{ns}}$ & $5134789466,2^{n}$ & $976134,5^{\mathrm{ns}}$ & $18097,8^{\mathrm{ns}}$ & $1753,9^{\mathrm{n}}$ & $653,8^{\mathrm{ns}}$ & $7784830599,9^{\mathrm{ns}}$ \\
\hline $\mathrm{G} \times \mathrm{A}$ & 87 & $89243,8 * *$ & $20666719,5^{* *}$ & $2707,3 * *$ & $39,5^{* *}$ & $18,7 * *$ & $4,2 * *$ & $44090616,2 * *$ \\
\hline Erro & 238 & 137491,0 & 29198481,9 & 6814,8 & 42,1 & 93,1 & 6,4 & 54287392,3 \\
\hline
\end{tabular}

Total 359

\begin{tabular}{llllllll}
\hline Média & 2811,7 & 21751,6 & 651,5 & 65,7 & 140,2 & 10,2 & 28378,7
\end{tabular}

** e * significativos a 1 e $5 \%$ de probabilidade, respectivamente, pelo teste $\mathrm{F}$; ns não-significativo, pelo teste $\mathrm{F}$. ** and * significant at 1 and $5 \%$ probability, respectively, by the F test; $n$ s non-significant, by the $F$ test.

Fonte: Autoria própria. Own authorship.

Tabela 2. Precipitação total anual (PrTotal, em $\mathrm{mm}$ ), médias das temperaturas máximas (TMax, em ${ }^{\circ} \mathrm{C}$ ), mínima (TMin, em ${ }^{\circ} \mathrm{C}$ ), temperatura média anual (TMed, em ${ }^{\circ} \mathrm{C}$ ) e média da UR\% do ar em Tomé-Açú, Belem, entre os anos de 2010 e 2012. Total annual precipitation (PrTotal, in mm), mean maximum temperatures average (TMax, in C), minimum temperatures average (TMin, in C), average annual temperature (TMed, in $C$ ) and $U R \%$ of the air average in Tomé -Açú, Belem, between the years 2010 and 2012.

\begin{tabular}{cccccc}
\hline & PrTotal & TMax & TMed & TMin & UR\% \\
\hline 2010 & 2203,8 & 33,4 & 26,5 & 21,5 & 85,0 \\
2011 & 3307,8 & 32,7 & 26,5 & 22,3 & 87,0 \\
2012 & 2438,5 & 32,5 & 26,4 & 22,2 & 85,0 \\
\hline
\end{tabular}

Fonte: Nilza Araújo Pacheco (meteorologista da estação de Tomé-Açú). Nilza Araújo Pacheco (meteorologist at Tomé-Açú station).

As variâncias da interação GxA $\left(\sigma^{2}\right.$ GxA $)$ foram negativas, o que indica que a intensidade dos efeitos residuais foi superior à da interação (CRUZ et al., 2012), apesar deste fato, com a estatística foi possível calcular os valores da interação GxA nas características avaliadas (Tabela 3). A variância ambiental foi superior a genética em todas as características, com isso, a maior parte das manifestações fenotípicas devem-se aos efeitos dos anos, resultado semelhante ao observado por Oliveira e Fernandes (2001), cujos efeitos residuais sempre foram superiores aos genotípicos.

Os coeficientes de determinação genotípico ou herdabilidade com base nas classes apresentadas por Resende (2009) seguiram a escala de consideração como baixos $\left(10<\mathrm{R}_{\mathrm{G}}^{2} \%\right.$ $<40)$, médios ou moderados $\left(40 \leq \mathrm{R}^{2}{ }_{\mathrm{G}} \% \leq 70\right)$ e altos $\left(\mathrm{R}_{\mathrm{G}}^{2} \%>70\right)$. Os coeficientes de determinação foram altos em PMCV e PCF, indicando que são facilmente reproduzidas em 
gerações posteriores, nestas condições ambientais e com o mesmo grupo de genótipos, enquanto as demais características, também podem reproduzir valores próximos, porém com menor precisão (Tabela 3). Estas herdabilidades foram responsáveis pela ausência dos efeitos ambientais observados na Tabela 1, também pelas diferenças significativas entre progênies detectadas em TMR e NTC.

Tabela 3. Parâmetros genéticos e fenotípicos das características peso médio do cacho (PMC), em gramas; peso total de frutos (PTF), em gramas; peso médio do cacho vazio (PMCV), em gramas; tamanho médio da ráquis/cacho (TMR), em centímetros; peso médio de 100 frutos (PCF), em gramas; número total de cachos (NTC) e; peso total do cacho (PTC), avaliadas em 30 progênies de açaizeiro (Euterpe oleracea). Genetic and phenotypic parameters of the characteristics bunch average weight (PMC); total fruit weight (PTF); average weight of empty bunch (PMCV); average size of the stem/bunch (TMR); average weight of 100 fruits $(P C F)$; total number of clusters (NTC) and; total weight of the bunch (PTC), evaluated in 30 progenies of assai palm trees.

\begin{tabular}{lrrrrrrr}
\hline Parâmetros $^{\text {a }}$ & \multicolumn{1}{c}{ PMC } & \multicolumn{1}{c}{ PTF } & PMCV & TMR & PCF & NTC & PTC \\
\hline$\sigma_{\text {GxA }}^{2}$ & $-15546,3$ & $-2749123,4$ & $-1323,5$ & 0,0 & $-24,0$ & $-0,7$ & $-3285627,9$ \\
$\sigma^{2}{ }_{\mathrm{A}}$ & 137491,0 & 29198481,9 & 6814,8 & 42,1 & 93,1 & 6,4 & 54287392,3 \\
$\sigma^{2}{ }_{\mathrm{G}}$ & 19999,4 & 4541341,2 & 1846,0 & 0,0 & 44,6 & 0,7 & 9110674,4 \\
$\mathrm{R}^{2} \%$ & 72,9 & 72,5 & 89,1 & 0,0 & 96,6 & 66,8 & 71,3 \\
$\mathrm{CVg}(\%)$ & 5,0 & 9,8 & 6,6 & - & 4,8 & 8,3 & 10,6 \\
$\mathrm{CVa}(\%)$ & 13,2 & 24,8 & 12,7 & 9,9 & 6,9 & 24,8 & 26,0 \\
$\mathrm{CVg} / \mathrm{CVa}$ & 0,38 & 0,39 & 0,52 & - & 0,69 & 0,33 & 0,41 \\
\hline
\end{tabular}

${ }^{\mathrm{a}} \sigma_{\mathrm{GxA}}^{2}$ : variância fenotípica da interação genótipos com anos; $\sigma_{\mathrm{A}}^{2}$ : variância ambiental com base na média para a característica; $\sigma^{2}{ }_{G}$ : variância genotípica com base na média para a característica; $\mathrm{R}^{2 \%}$ : coeficiente de determinação genotípico ou herdabilidade no sentido amplo em porcentagem; $\operatorname{CVg}(\%)$ : coeficiente de variação genotípica em porcentagem; CVe (\%): coeficiente de variação ambiental em porcentagem; CVg/CVa: relação entre coeficiente de variação genotípica pelo coeficiente de variação ambiental. ${ }^{a} \sigma_{G x A}^{2}$ : phenotypic variance of interaction from genotype with environments; $\sigma_{A}^{2}$ : environmental variance based on the average for the characteristic; $\sigma_{G}^{2}$ :genotypic variance based on the average for the characteristic; $R^{2} \%$ : genotypic determination coefficient or heritability in the broad sense in percentage; $\mathrm{CVg}(\%)$ : genotype coefficient of variation in percentage; CVa (\%): coefficient of environmental variation in percentage; CVg/CVa: relation between genotype coefficient of variation by coefficient of environmental variation.

Fonte: Autoria própria. Own authorship.

Em materiais genéticos onde é desejado a seleção, espera-se encontrar valores de $\mathrm{CVg}$ relacionados ao genótipo, superiores ao $\mathrm{CVe}$, que estão associados às variações ambientais, na Tabela 3 tem-se que em todas as características o CVg foi inferior ao CVe. Para facilitar a visualização do comportamento dos materiais genético é utilizado um índice que serve como indicativo da facilidade em se realizar a seleção e obter resultados, que é a relação entre o coeficiente de variação genético e o residual, sendo que valores próximos ou acima da unidade demonstram grande chance em se obter sucesso com menores esforços. As duas melhores relações de $\mathrm{CVg} / \mathrm{CVe}$ foram para as características PMCV e PCF, contudo não foram superiores o 0,70 , indicando que a variabilidade genética disponível pode restringir e dificultar a seleção. 
Tabela 4. Estimativas do coeficiente de repetibilidade $(\hat{\rho})$, coeficiente de determinação $\left(\mathrm{R}^{2}\right)$ e número de medições ${ }^{1}(\eta)$ calculado para as características peso médio do cacho (PMC), em gramas; peso total de frutos (PTF), em gramas; peso médio do cacho vazio (PMCV), em gramas; tamanho médio da ráquis/cacho (TMR), em centímetros; peso médio de 100 frutos (PCF), em gramas; número total de cachos (NTC) e; peso total do cacho (PTC), avaliadas em 30 progênies de açaizeiros, obtidas pelos métodos da Análise de Variância (ANOVA), Componentes Principais baseados na Matriz de Covariâncias (CPCOV) e de Correlações (CPCOR) e, Análise Estrutural baseado na Matriz de Correlações (AECOR) e na Matriz de Covariância (AECOV). Estimates of the repeatability coefficient ( $\hat{\rho})$, coefficient of determination (R2) and number of measurements 1 ( $\eta)$ calculated for characteristics bunch average weight (PMC); total fruit weight (PTF); average weight of empty bunch (PMCV); average size of the stem/bunch (TMR); average weight of 100 fruits $(P C F)$; total number of clusters (NTC) and; total weight of the bunch (PTC), evaluated in 30 progenies of assai palm trees, obtained by the methods of Analysis of Variance ANOVA), Principal Components based on the Covariance Matrix (CPCOV) and Correlations Matrix (CPCOR) and, Structural Analysis based on the Correlation Matrix (AECOR) and the Covariance Matrix (AECOV).

\begin{tabular}{|c|c|c|c|c|c|c|}
\hline \multirow[b]{2}{*}{ Caráter } & \multirow[b]{2}{*}{ Estimativa } & \multicolumn{5}{|c|}{ Método } \\
\hline & & ANOVA & CPCOV & CPCOR & AECOR & AECOV \\
\hline \multirow[t]{4}{*}{ PMC } & $\hat{\rho}$ & 0,402 & 0,546 & 0,406 & 0,390 & 0,402 \\
\hline & $\mathrm{R}^{2} \%$ & 72,89 & 82,74 & 73,21 & 71,88 & 72,89 \\
\hline & $\eta 1$ & 8,43 & & 4,72 & 8,30 & 8,69 \\
\hline & $\eta 2$ & 28,26 & & 15,82 & 27,81 & 29,73 \\
\hline \multirow[t]{4}{*}{ PTF } & $\hat{\rho}$ & 0,397 & 0,482 & 0,452 & 0,405 & 0,397 \\
\hline & $\mathrm{R}^{2} \%$ & 72,50 & 78,83 & 76,74 & 73,11 & 72,50 \\
\hline & $\eta 1$ & 8,60 & & 6,09 & 6,87 & 8,34 \\
\hline & $\eta 2$ & 28,82 & & 20,41 & 23,03 & 27,96 \\
\hline \multirow[t]{4}{*}{ PMCV } & $\hat{\rho}$ & 0,672 & 0,746 & 0,695 & 0,693 & 0,672 \\
\hline & $\mathrm{R}^{2} \%$ & 89,11 & 92,16 & 90,12 & 90,02 & 89,11 \\
\hline & $\eta 1$ & 2,77 & & 1,93 & 2,48 & 2,51 \\
\hline & $\eta 2$ & 9,29 & & 6,47 & 8,33 & 8,42 \\
\hline \multirow[t]{4}{*}{ TMR } & $\hat{\rho}$ & 0,099 & 0,398 & 0,294 & 0,201 & 0,099 \\
\hline & $\mathrm{R}^{2} \%$ & 30,57 & 72,55 & 62,537 & 50,97 & 30,57 \\
\hline & $\eta 1$ & 51,47 & & 8,58 & 13,58 & 21,80 \\
\hline & $\eta 2$ & 172,58 & & 28,76 & 45,53 & 73,10 \\
\hline \multirow[t]{4}{*}{ PCF } & $\hat{\rho}$ & 0,877 & 0,889 & 0,884 & 0,884 & 0,877 \\
\hline & $\mathrm{R}^{2} \%$ & 96,62 & 96,97 & 96,84 & 96,83 & 96,62 \\
\hline & $\eta 1$ & 0,79 & & 0,71 & 0,74 & 0,74 \\
\hline & $\eta 2$ & 2,66 & & 2,37 & 2,48 & 2,49 \\
\hline \multirow[t]{4}{*}{ NTC } & $\hat{\rho}$ & 0,335 & 0,513 & 0,352 & 0,288 & 0,335 \\
\hline & $\mathrm{R}^{2 \%}$ & 66,83 & 80,84 & 68,47 & 61,75 & 66,83 \\
\hline & $\eta 1$ & 11,25 & & 5,37 & 10,44 & 14,04 \\
\hline & $\eta 2$ & 37,73 & & 18,01 & 35,01 & 47,08 \\
\hline
\end{tabular}


Tabela 4. Continuação...

\begin{tabular}{llccccc}
\hline & \multicolumn{5}{c}{ Método } \\
\hline Caráter & Estimativa & ANOVA & CPCOV & CPCOR & AECOR & AECOV \\
\hline PTC & $\hat{\rho}$ & 0,383 & 0,534 & 0,457 & 0,401 & 0,383 \\
& $\mathrm{R}^{2} \%$ & 71,26 & 82,07 & 77,06 & 72,82 & 71,26 \\
& $\eta 1$ & 9,14 & & 4,95 & 6,75 & 8,46 \\
& $\eta 2$ & 30,65 & & 16,60 & 22,62 & 28,37 \\
\hline
\end{tabular}

${ }^{1} \eta 1$ e $\eta 2$, número de medições desejável para predizer o valor real dos indivíduos com 85 e $95 \%$ de determinação, respectivamente. ${ }^{1} \eta 1$ e $\eta 2$, number of measurements to predict the real value of individuals with 85 and $95 \%$ determination, respectively.

Fonte: Autoria própria. Own authorship.

$\mathrm{Na}$ Tabela 4 é apresentada a análise de repetibilidade $(\hat{\rho})$, os coeficientes de determinação genotípico $\left(R^{2}\right)$ e o número de medições $(\mathrm{y})$ necessárias à determinação mais exata dos genótipos. Valores baixos de repetibilidade indicam que a expressão possui elevada contribuição ambiental, cujo comportamento não pode ser predito. Enquanto, valores altos indicam maiores estabilidades devido à maior contribuição de origem genética em relação a ambiental (RESENDE, 2009).

Foram considerados como coeficientes de repetibilidade baixos e inexpressivos valores inferiores a 0,3, conforme Resende (2009), com indícios da inexistência de regularidade nas sucessivas avaliações. Desta forma a característica TMR em todas as metodologias, exceto CPCOV, teve baixa repetibilidade, com indicativo de que existe maior contribuição de efeitos da interação GxA na manifestação fenotípica e, portanto, em todo novo local é difícil predizer o comportamento destes genótipos, o mesmo ocorreu para NTC na metodologia AECOR. Nos diversos trabalhos existentes não houve característica com coeficiente de repetibilidade baixo, tanto em frutos de macaúba por Manfio et al. (2011), puçá coroa de frade por Silveira et al. (2014) e, em frutos de juçara por Marçal et al. (2016), esta diferença possivelmente ocorreu devido ao açaizeiro estar ainda em processo de domesticação.

Valores superiores a 0,6 para os coeficientes de repetibilidade foram considerados como altos, conforme Resende (2009), cuja expressão fenotípica em sua maioria é proveniente de controle genético. Aqui as características PMCV e PCF apresentaram valores altos, indicando que houve alta repetibilidade nas sucessivas avaliações. O peso de cem frutos também apresentou valor alto em Oliveira e Fernandes (2001), apesar de ter tido estimativa inferior (0,63). Nos diversos trabalhos como em bacuri por Silva et al. (2009), em frutos de macaúba por Manfio et al. (2011), em puçá coroa de frade por Silveira et al. (2014), em frutos de juçara obtidos por Marçal et al. (2016), há ampla variação, indicando que qualquer modificação nos fatores ambientais ou no pool genético da espécie estudada, os valores se alteram, ressaltando que, nenhum dos estudos apresentou estimativa considerada baixa, demonstrando elevado controle genético.

As características PMCV e PCF, podem ser utilizadas como parâmetros desejáveis em processos simples de melhoramento para o açaizeiro, a exemplo da seleção fenotípica simples, com perspectivas de se obter bom ganho genético. Nas características que apresentaram coeficientes de repetibilidade baixos, sendo bastante influenciados pelos 
diferentes anos, sugere-se que, no emprego dessas variáveis, sejam propostos métodos de melhoramento com o máximo de controle ambiental e delineamentos experimentais mais robustos, pois aqui no presente estudo a seleção massal seria ineficiente. As características PMC, PTF, PTC em todas as metodologias apresentaram estimativas médias, somando-se a TMR na metodologia CPCOV e NTC, diferindo ao citado por Oliveira e Fernandes (2001) em PTF e PTC que obtiveram valores baixos.

Um aspecto observado nas estimativas de repetibilidade em plantas perenes pelo método da ANOVA e por métodos multivariados (análise estrutural e componentes principais), como em frutos de maracujá por Neves et al. (2010) e Santos et al. (2010), em cirigueleiras por Lira Júnior et al. (2014) foi de que os valores obtidos pelo método da ANOVA alcançaram resultados sempre inferiores às obtidas pela análise multivariada. Contudo este comportamento não ocorreu nas análises da Tabela 4, pois a metodologia ANOVA apesar de apresentar valores menores mostrou-se próxima àquelas obtidas pelo método CPCOR, análogo ao notado na produção de cachos de híbridos entre caiaué e dendezeiro por Chia et al. (2009), em frutos de macaúba por Manfio et al. (2011) e em pêssegos por Matias et al. (2014) onde os valores foram menores, contudo próximos, ou iguais aos obtidos por CPCOR. Já em bananeiras por Yokomizo e Azevedo Dias (2016) os valores foram maiores na metodologia ANOVA. Uma explicação da inferioridade observada em geral pelo método de ANOVA seria uma resposta decorrente da variância genotípica, utilizada para estimar a repetibilidade não ser proveniente apenas da parte genética, uma vez que o componente de variância ambiental permanente entre indivíduos permanece confundido com a variância genotípica (CHIA et al., 2009).

Os coeficientes de determinação genotípico para as características avaliadas foram considerados altos, com valores acima de $70 \%$ e, conforme Resende (2009), baseado neste limite, PMC, PTF, PMCV, PCF e PTC, foram consideradas confiáveis em todas as metodologias, apesar das estimativas terem sido menores que os citados por Santos et al. (2010) em maracujazeiros, por Matias et al. (2014) em pêssegos e em frutos de juçara por Marçal et al. (2016), e dentro das amplitudes obtidas em maracujazeiro por Neves et al. (2010), em frutos de macaúba por Manfio et al. (2011), por Lira Júnior et al. (2014) em cirigueleiras e Yokomizo e Azevedo Dias (2016) em bananeiras. Já em TMR e NTC nas diferentes metodologias, excetuando-se em CPCOV, não ofereceram respaldo quanto ao número de medições necessárias, tendo-se que efetuar avaliações adicionais.

Em vista desses resultados, o número de medições desejáveis para uma seleção mais efetiva nas características PMC, PTF, TMR, NTC e PTC, com um coeficiente de determinação de $95 \%$, foi entre 15,82 a 172,58, resumindo-se em baixa viabilidade prática, dificultando consideravelmente suas aplicações na seleção de genótipos desejáveis nas condições do Pará, elevando em demasia a quantidade de avaliações a serem obtidas no campo, sendo que Neves et al. (2010) em maracujazeiro obtiveram valores mais próximos do quantitativo menor de medições aqui observadas, enquanto que Santos et al. (2010), Lira Júnior et al. (2014) e, Yokomizo e Azevedo Dias (2016) estimaram valores entre 6 a 33 medições, diferindo do que foi observado aqui. 
Essa dificuldade na quantidade de medições necessárias foi citada também na produção de cachos de híbridos entre caiaué e dendezeiro por Chia et al. (2009), em frutos de bacuri por Silva et al. (2009), em puçá coroa de frade no trabalho de Silveira et al. (2014) com até 22 medições. Já comparativamente ao de Marçal et al. (2016) em frutos de juçara, somente poucas medições foram suficientes, semelhante ao que ocorreu nas características PMCV e PCF.

Na característica PMCV o número de medições entre 6,47 a 9,29, e para PCF com valores entre 2,37 e 2,66, estão mais próximas de um processo factível, situando-se dentro dos limites obtidos em frutos de pêssego por Matias et al. (2014) e comparativamente PCF foi semelhante ao quantitativo obtido nas avaliações de frutos de macaúba obtido por Manfio et al. (2011).

Considerando-se um coeficiente de determinação de $85 \%$, tem-se um quantitativo de medições necessárias entre 4,72 e 14,84, para predição do comportamento das progênies nas características PMC, PTF, NTC e PTC, mais factível na prática, estando proporcionalmente semelhante a aqueles citados por Chia et al. (2009) em médias de progênies de híbridos entre caiaué e dendezeiro para produção de cachos e de Neves et al. (2010) em maracujazeiro. A característica TMR ainda apresentou valores entre 8,58 a 51,47. Estes dados indicam que há a necessidade de um grande quantitativo de número de avaliações para se ter uma predição alta do comportamento deste grupo de genótipos, assim como também ocorreu em indivíduos de híbridos entre caiaué e dendezeiro para produção de cachos obtido por Chia et al. (2009), enquanto que a necessidade de apenas uma medição em frutos de macaúba obtido por Manfio et al. (2011) assemelhou-se as das características PMCV e PCF que mostraram valores necessários abaixo das quatro avaliações realizadas.

A possível diferença entre os resultados obtidos por Oliveira e Fernandes (2001) é que estes autores avaliaram quatro cachos por planta dentro do mesmo ano, enquanto que as análises aqui apresentadas foram cachos de quatro anos, ou seja, no mesmo ano há a tendência de variabilidade nas características relativas à produção, enquanto que entre anos ocorre maior uniformidade.

Os limites adotados nas classes de correlações foi a citada por Santos (2018), considerando correlações entre 0 a $10 \%$ como baixas; 10 a $50 \%$ como médias baixas; 50 a $80 \%$ como médias altas e acima de $80 \%$ como altas.

Visando facilitar a seleção de progênies com baixa repetibilidade em TMR e NTC, principalmente nesta segunda característica, visualizou-se a possibilidade de utilizar a correlação positiva com outra característica que possuísse alta repetibilidade, para a realização de uma seleção indireta. Na Tabela 5 as correlações entre TMR e PMCV com PCF foram negativas, indicando que com maior tamanho do cacho em peso e em comprimento os frutos produzidos serão menores; também ocorreu correlação negativa entre NTC e PMCV, com tendência de maior peso do cacho vazio gerar menor número de cachos.

O TMR teve correlação genética alta com o peso médio do cacho (PMC), indicando que a presença de ráquis mais comprida tem-se maior o peso do cacho com frutos, resultado importante por permitir a seleção indireta de TMR por PMC, associado ao aspecto da 
correlação ter sido média alta também com PMCV e PTF, demonstrando o mesmo comportamento. $\mathrm{O}$ único inconveniente é que PMC apresentou média, tornando difícil a seleção indireta de TMR.

Tabela 5. Correlações genotípicas entre as características peso médio do cacho (PMC), em gramas; peso total de frutos (PTF), em gramas; peso médio do cacho vazio (PMCV), em gramas; tamanho médio da ráquis/cacho (TMR), em centímetros; peso médio de 100 frutos (PCF), em gramas; número total de cachos (NTC) e; peso total do cacho (PTC), avaliadas em 30 progênies de açaizeiro. Genotypic correlations between the characteristics bunch average weight (PMC); total fruit weight (PTF); average weight of empty bunch (PMCV); average size of the stem/bunch (TMR); average weight of 100 fruits (PCF); total number of clusters (NTC) and; total weight of the bunch (PTC), evaluated in 30 progenies of assai palm trees

\begin{tabular}{ccccccc}
\hline & PTF & PMCV & TMR & PCF & NTC & PTC \\
\hline PMC & 75,14 & 64,41 & 93,95 & 18,53 & 38,15 & 74,82 \\
PTF & - & 15,71 & 72,96 & 48,62 & 88,09 & 98,84 \\
PMCV & & - & 100,00 & $-9,94$ & $-29,30$ & 31,76 \\
TMR & & & - & $-15,20$ & 50,52 & 86,37 \\
PCF & & & & - & 55,96 & 45,46 \\
NTC & & & & & - & 88,92 \\
\hline
\end{tabular}

Fonte: Autoria própria. Own authorship.

O peso total de frutos (PTF) teve correlações média alta com TMR e alta com NTC, indicando que a produtividade está associada positivamente com o tamanho da ráquis e o quantitativo de cachos, mas também neste caso a seleção indireta utilizando-se PTF não apresenta condições favoráveis já que sua repetibilidade é média.

Houve correlação média alta entre NTC e PCF, em que plantas que produzem número maior de cachos também tem os frutos maiores. Já entre TMR e PMCV foi total indicando que quanto maior o comprimento da ráquis com certeza o cacho vazio será mais pesado, sendo conveniente ressaltar que tanto PCF como PMCV possuem repetibilidade alta, mas infelizmente não tiveram altas correlações com ambas as características (TMR e NTC).

Em avaliações de correlações Oliveira e Fernandes (2001) também encontraram comportamento semelhante entre PTC e PTF; PTC e PCF e; PTF e PCF, que mostra a forte relação entre características de cachos com a produção e tamanho de frutos.

Desta forma, para a seleção indireta, em características com baixa repetibilidade, por outra característica que possua alta repetibilidade e correlação positiva simultaneamente com TMR e NTC. Nestas condições, com alta repetibilidade, PCF e PMCV apresentaram respectivamente correlações negativas com TMR e NTC. Com PMC houve correlação média baixa com NTC. As características PTF e PTC apresentaram correlações altas com NTC e TMR, contudo possuem repetibilidade média, ou seja, utilizando-se as correlações é difícil a seleção simultânea e indireta. Devendo-se optar por realizar de forma indireta e particularizada, ou seja, uma opção seria NTC com PCF e entre TMR com PMCV. 


\section{CONCLUSÕES}

Os efeitos isolados dos anos não foram suficientes para gerar diferenças nas características, contudo a interação GxA diferenciada foi presente e gerou diferenças, o que significa que as diferenças genéticas existentes nas progênies interagem de forma diferenciada com os fatores ambientais.

Todas as características relacionadas a peso apresentaram herdabilidades e repetibilidades altas, facilitando uma seleção futura com estas características, enquanto em TMR e NTC há dificuldades devido à incerteza de comportamento e necessidade de um grande número de avaliações dificultando os trabalhos de seleção.

Uma solução para as pesquisas com TMR e NTC está apoiada nos valores de correlações, altas e positivas com PTC, podendo se utilizar está característica em substituição as duas.

Baseado no número de medições desejáveis na análise de repetibilidade, as características PTC, PMC e PTF, excluindo-se TMR e NTC, necessitam de maior número de avaliações ainda, para conseguir uma predição com maior precisão sobre seus comportamentos. Recomenda-se, portanto, somente realizar uma seleção para esta população quando se alcançar o número de medições determinados pela análise de repetibilidade.

O tamanho de fruto independe das demais características, facilitando sua seleção, pois há mercados que preferem frutos grandes e outros que preferem frutos pequenos.

\section{REFERÊNCIAS BIBLIOGRÁFICAS}

CHIA, G. S.; LOPES, R.; CUNHA, R. N. V.; ROCHA, R. N. C.; LOPES, M. T. G. Repetibilidade da produção de cachos de híbridos interespecíficos entre o caiaué e o dendezeiro. Acta Amazonica, Manaus, v. 39, n. 2, p.249-254, 2009.

CRUZ, C. D. GENES - a software package for analysis in experimental statistics and quantitative genetics. Acta Scientiarum, Maringá, v. 35, n. 3, p.271-276, 2013.

CRUZ, C. D.; REGAZZI, A. J.; CARNEIRO, P. C. S. Modelos biométricos aplicados ao melhoramento genético. Viçosa: UFV, 2012. v. 1, 514 p.

FARIAS NETO, J. T.; RESENDE, M. D. V.; OLIVEIRA, M. S. P.; NOGUEIRA, O. L.; FALCÃO, P. N. B.; SANTOS, N. S. A. Estimativas de parâmetros genéticos e ganho de seleção em progênies de polinização aberta de açaizeiro. Revista Brasileira de Fruticultura, Jaboticabal, v. 30, n. 4, p.1051-1056, 2008.

INSTITUTO BRASILEIRO DE GEOGRAFIA E ESTATÍSTICA - IBGE. Produção da Extração Vegetal e da Silvicultura 2017. Rio de Janeiro: IBGE, 2017. v. 32, 7 p.

LIRA JUNIOR, J. S.; BEZERRA, J. E. F.; MOURA, R. J. M.; SANTOS, V. F.Repetibilidade da produção, número e peso de fruto em cirigueleira (Spondias purpurea L.). Revista Brasileira de Fruticultura, Jaboticabal, v. 36, n. 1, p.214-220, 2014.

LUSH, J. L. Animal breeding plans, 4. ed. Olney: Orchard Press, 2008. 444 p. 
MAIA, M. C. C.; RESENDE, M. D. V.; PAIVA, J. R.; CAVALCANTI, J. J. V.; BARROS, L. M. Seleção simultânea para produção, adaptabilidade e estabilidade genotípicas em clones de cajueiro, via modelos mistos. Pesquisa Agropecuária Tropical, Goiânia, v. 39, n. 1, p.43$50,2009$.

MANFIO, C. E.; MOTOIKEI, S. Y.; SANTOS, C. E. M.; PIMENTEL, L. D.; QUEIROZ, V.; SATOI, A. Y. Repetibilidade em características biométricas do fruto de macaúba. Ciência Rural, Santa Maria, v. 41, n. 1, p.70-76, 2011.

MARÇAL, T. S; GUILHEN, J. H. S; OLIVEIRA, W. B. S; FERREIRA, M. F. S.; RESENDE, M. D. V.; FERREIRA, A. Repetibilidade de características biométricas de frutos de palmito Juçara. Bioscience Journal, Uberlândia, v. 32, n. 4, p.890-898, 2016.

MATIAS, R. G. P.; BRUCKNER, C. H.; CARNEIRO, P. C. S.; SILVA, D. F. P.; SILVA, J. O. C. Repeatability, correlation and path analysis of physical and chemical characteristics of peach fruits. Revista Brasileira de Fruticultura, Jaboticabal, v. 36, n. 4, p.971-979, 2014.

NEVES, L. G; BRUCKNER, C. H.; CRUZ, C. D.; BARELL, M. A. A. Avaliação da repetibilidade no melhoramento de famílias de maracujazeiro. Revista Ceres, Viçosa, v. 57, n. 4, p.480-485, 2010.

NOGUEIRA, A. K. M.; SANTANA, A. C. Benefícios socioeconômicos da adoção de novas tecnologias no cultivo do açaí no Estado do Pará. Revista Ceres, Viçosa, v. 63, n. 1, p.1-7, 2016.

NOGUEIRA, A. K. M.; SANTANA, A. C.; GARCIA, W. S. A dinâmica do mercado de açaí fruto no Estado do Pará: de 1994 a 2009. Revista Ceres, Viçosa, v. 60, n. 3, p.324-331, 2013.

OLIVEIRA, M. S. P.; FERNANDES, G. L. C. Repetibilidade de caracteres do cacho de açaizeiro nas condições de Belém-PA. Revista Brasileira de Fruticultura, Jaboticabal, v. 23, n. 3, p.613-616, 2001.

QUEIRÓZ, J. A. L.; MOCHIUTTI, S.; BIANCHETTI, A. Produção de mudas de açaí. Macapá: Embrapa Amapá, 2001. 6 p. (Embrapa Amapá. Comunicado técnico, 54).

RESENDE, M. D. V. Genética biométrica e estatística no melhoramento de plantas perenes. Brasília: Embrapa Informação Tecnológica, 2009. 975 p.

SANTOS, C. E. M.; BRUCKNER, C. H.; CRUZ, C. D.; SIQUEIRA, D. L.; PIMENTEL, L. D.; ROSADO, L. D. S. Repetibilidade em características do fruto do maracujazeiro. Revista Ceres, Viçosa, v. 57, n. 3, p.343-350, 2010.

SANTOS, C. Estatística Descritiva - Manual de Auto-aprendizagem. 3. ed. Lisboa: Edições Silabo, 2018. 310 p.

SILVA, R. G.; CHAVES, M. C. L.; ARNHOLD, E.; CRUZ, C. D. Repetibilidade e correlação fenotípicas de caracteres do fruto de bacuri no estado do Maranhão. Acta Scientiarum Agronomy, Maringá, v. 31, n. 4, p.587-591, 2009.

SILVEIRA, M. R. S.; ALVES, R. E.; ARAGAO, F. A. S.; FIGUEIREDO, R. W.; FREITAS, S. L. A. Estudo de genótipos de puçá coroa de frade da vegetação litorânea de Beberibe-CE. Revista Caatinga, Mossoró, v. 27, n. 2, p.159-165, 2014. 
VAlEnTE, M. A.; WATRIN, O. S.; CASTRO, A. R. C. Mapeamento Detalhado dos Solos da Fazenda Experimental da Embrapa Amazônia Oriental em Tomé-Açu, PA. Belém: Embrapa Amazônia Oriental, 2014. 33 p. (Documentos, 405).

YOKOMIZO, G. K.; AZEVEDO DIAS, J. S. Repetibilidade e correlações entre caracteres morfo-agronômicos em bananeiras no Amapá. Scientia Rural, Ponta Grossa, v. 1, n. 14, p.94-105, 2016. 